\title{
APPROXIMATION BY p-ADIC LIE GROUPS
}

\author{
HELGE GLÖCKNER \\ Technische Universität Darmstadt, Fachbereich Mathematik AG 5 , \\ Schloßgartenstr. 7, 64289 Darmstadt, Germany \\ e-mail: gloeckner@mathematik.tu-darmstadt.de
}

(Received 5 July, 2000; accepted 6 September 2000)

\begin{abstract}
We discuss classes of topological groups which can be approximated by $p$-adic Lie groups, and varieties of Hausdorff groups generated by classes of $p$-adic Lie groups (for a single or multiple $p$ ). We give several characterizations of locally compact pro- $p$-adic Lie groups and locally compact pro-discrete groups, and prove a "pro-version" of Cartan's Theorem: whenever a locally compact group is a pro- $p$-adic Lie group and a pro- $q$-adic Lie group for distinct primes $p$ and $q$, it is pro-discrete. If a locally compact group can be approximated by $p$-adic Lie groups for variable primes $p$, then it is a pro- $p$-adic Lie group for some prime $p$.
\end{abstract}

2000 Mathematics Subject Classification. Primary 22D05. Secondary 20E26, 14L10.

Introduction. Every connected, locally compact group $G$ can be approximated by real Lie groups [15], a fact which is of crucial importance in the structure theory of locally compact groups. In contrast, it is known that totally disconnected, locally compact groups cannot be approximated by Lie groups over (totally disconnected) local fields in general [17]: the continuous homomorphisms into Lie groups over local fields need not even separate points (Theorem 5.2 below). Still, Lie groups over local fields, at least $p$-adic Lie groups, do allow fruitful applications in the study of totally disconnected, locally compact groups, as long as one restricts one's studies to special classes of these groups. The aim of this paper is to describe and investigate such classes, or prospective candidates.

Having assembled the preliminaries in Section 1, we give various characterizations of locally compact pro- $p$-adic Lie groups. In particular, a locally compact group is a pro- $p$-adic Lie group if and only if it is a member of the variety of Hausdorff groups generated by the class of $p$-adic Lie groups. Similar characterizations can be obtained for locally compact pro-discrete groups, and more generally for locally compact pro-A-groups, where $\mathbb{A}$ is an "admissible property of topological groups" (Theorem 2.1). The latter concept has been introduced here to facilitate a unified discussion of the classes of groups just mentioned, along the lines of the treatment of pro-Lie groups given in [11].

It turns out that a locally compact group which is both a pro-p-adic Lie group and a pro- $q$-adic Lie group, for distinct primes $p$ and $q$, has to be pro-discrete (Theorem 3.1). A locally compact group which can be approximated by $p$-adic Lie groups, with $p$ varying within a certain set of primes $\mathfrak{p}$, is a pro- $p$-adic Lie group for some $p \in \mathfrak{p}$ (Theorem 4.1).

In view of the characterization of locally compact pro- $p$-adic Lie groups mentioned before, it appears most natural to consider also the classes $\mathbb{M} \llbracket \mathbb{X}_{\mathfrak{p}}$ of those totally 
disconnected, locally compact groups which can be built up from $p$-adic Lie groups, where $p$ varies within a fixed set of primes $\mathfrak{p}$, i.e., which are contained in the variety of Hausdorff groups generated by the $p$-adic Lie groups, with $p \in \mathfrak{p}$. We show that there exist totally disconnected, locally compact groups which are not members of $\mathbb{M} \square \mathbb{X}_{\mathbb{P}}$ (where $\mathbb{P}$ is the set of all primes). There is even a compactly generated counter example (Theorem 5.2).

The article concludes with further examples, open problems, and a short glimpse of results specific to the approximation of compactly generated locally compact groups by $p$-adic Lie groups. Further applications of $p$-adic Lie theory in the study of totally disconnected, locally compact groups might be facilitated by the consideration of the $p$-adic Lie algebras of these groups, as defined in [6].

1. Notation and Terminology. Throughout this article, $\mathbb{T} \mathbb{G}$ denotes the category of Hausdorff topological groups and continuous homomorphisms between them ("morphisms" for short).

Let $\mathbb{A}$ be a class of Hausdorff groups which contains the trivial group and is closed under passage to isomorphic topological groups: then $\mathbb{A}$ is called a property of topological groups; the elements of $\mathbb{A}$ are called $\mathbb{A}$-groups [9, Definition 1.17].

1.1 If $G$ is a Hausdorff group, we let $\operatorname{Hom}(G, \mathbb{A})$ denote the class of all morphisms $f: G \rightarrow H$ such that $H \in \mathbb{A}$, and we let $\mathrm{N}_{\mathbb{A}}(G)$ be the set of all closed normal subgroups $N$ of $G$ such that $G / N$ is an A-group. We say that $G$ is a residual A-group if $\operatorname{Hom}(G, \mathbb{A})$ separates points on $G$; we say that $G$ can be approximated by A-groups if for every identity neighbourhood $U$ in $G$, there exists $N \in \mathrm{N}_{\mathrm{A}}(G)$ such that $N \subseteq U$.

1.2 If $G$ is a Hausdorff group with the property that $\mathrm{N}_{A}(G)$ is a filter basis, we associate a projective limit of $\mathbb{A}$-groups $G_{\mathbb{A}}$ to $G$, as follows: we make $\mathcal{N}:=\mathrm{N}_{\mathbb{A}}(G)$ a directed set by means of inverse inclusion as the ordering, i.e., $N \preceq M \Leftrightarrow N \supseteq M$, and let $q_{N M}: G / M \rightarrow G / N$ denote the map obtained by factoring the canonical quotient morphism $q_{N}: G \rightarrow G / N$ through $q_{M}$ for $N \preceq M$. Then $\left((G / N),\left(q_{N M}\right)\right)$ is a projective system. We set

$$
G_{\mathbb{A}}:=\lim _{\leftarrow \in \mathcal{N}} G / N \leq \prod_{N \in \mathcal{N}} G / N
$$

and we let $\eta_{\mathbb{A}}: G \rightarrow G_{\mathbb{A}}$ be the unique morphism such that $\pi_{N} \circ \eta_{\mathbb{A}}=q_{N}$ for every $N \in \mathcal{N}$, where $\pi_{N}:=\left.\operatorname{pr}_{N}\right|_{G_{\AA}}: G_{\AA} \rightarrow G / N$ denotes the respective limit map.

1.3 Note that $\eta_{\mathbb{A}}$ has dense image: if $y=\left(y_{N}\right)_{N \in \mathcal{N}} \in G_{\mathbb{A}}$ and $U$ is a neighbourhood of $y$ in $G_{\AA}$, there exist a finite subset $\mathcal{F}$ of $\mathcal{N}$ and open neighbourhoods $V_{N}$ of $y_{N}$ in $G / N$ such that $G_{\mathbb{A}} \cap \prod_{N \in \mathcal{N}} V_{N} \subseteq U$ and $V_{N}=G / N$ whenever $N \notin \mathcal{F}$. Since $\mathcal{N}=\mathrm{N}_{\mathrm{A}}(G)$ is a filter basis, there exists $N_{0} \in \mathcal{N}$ such that $N_{0} \subseteq \bigcap \mathcal{F}$. Then $U_{0}:=\pi_{N_{0}}^{-1}(V)$, where $V:=\bigcap_{N \in \mathcal{F}} q_{N N_{0}}^{-1}\left(V_{N}\right)$, is a neighbourhood of $y$ contained in $U$. Now $y_{N_{0}}=q_{N_{0}}(x)$ for some $x \in G$, and $\pi_{N_{0}}\left(\eta_{A}(x)\right)=y_{N_{0}}$ shows that $\eta_{\mathbb{A}}(x) \in U_{0} \subseteq U$.

1.4 Recall that a Hausdorff group $G$ is called a pro-A-group if the following conditions are satisfied [9, Definition 1.17]:

(I) $\mathrm{N}_{\mathbb{A}}(G)$ is a filter basis which converges to 1 in $G$; 
(II) for every morphism $\psi: G \rightarrow H$ into an A-group $H$, the quotient group $G / \operatorname{ker} \psi$ is an $\mathbb{A}$-group;

(III) $G$, together with the quotient maps $q_{N}: G \rightarrow G / N$, is a projective limit in $\mathbb{T} \mathbb{G}$ of the projective system $\left((G / N)_{N \in \mathrm{N}_{A}(G)},\left(q_{N M}\right)\right)$ described above. [In view of (I), this condition is equivalent to $\eta_{\mathbb{A}}: G \rightarrow G_{\mathbb{A}}$ being an isomorphism of topological groups.]

Having introduced our terminology concerning approximation by $\mathbb{A}$-groups, we turn to topological groups which can be "built up" from A-groups.

1.5 A class $\mathcal{W}$ of Hausdorff groups is called a variety of Hausdorff groups if $\mathcal{W}$ is closed under the operations of forming subgroups (S), quotient groups with respect to closed normal subgroups $(\overline{\mathrm{Q}})$, arbitrary cartesian products $(\mathrm{C})$ (and under passage to isomorphic topological groups). See [11]. If $\mathbb{A}$ is a class of Hausdorff groups, there is a smallest variety $\mathcal{V}(\mathbb{A})$ of Hausdorff groups such that $\mathbb{A}$ is a subclass of $\mathcal{V}(\mathbb{A})$. If, in addition to the operations above, $(\mathrm{P})$ denotes the formation of finite cartesian products and $(\overline{\mathrm{S}})$ the formation of closed subgroups, it can be shown that

$$
\mathcal{V}(\mathbb{A})=\operatorname{SC} \overline{\mathrm{Q}} \overline{\mathrm{SP}}(\mathbb{A})
$$

See [4, Theorem 2].

1.6 We call a property $\mathbb{A}$ of topological groups an admissible property of topological groups if $\mathbb{A}=\bar{Q} \bar{S} P(\mathbb{A})$, if every $\mathbb{A}$-group is locally compact, and if $G / \operatorname{ker} f$ is an $\mathbb{A}$-group, for every morphism $f: G \rightarrow H$ from a locally compact group $G$ into an A-group $H$. Then simply $\mathcal{V}(\mathbb{A})=\operatorname{SC}(\mathbb{A})$, and $\mathrm{N}_{\mathbb{A}}(G)$ is a filter basis, for every locally compact group $G$.

1.7 Note that Hausdorff quotients, finite products, and closed subgroups of $p$-adic Lie groups are also $p$-adic Lie groups. Further, if $f: G \rightarrow H$ is a morphism from a locally compact group $G$ into a $p$-adic Lie group, then $G / \operatorname{ker} f$ is a $p$-adic Lie group by $[3, \S 8.2$, Corollary 1 to Theorem 2]. Hence the $p$-adic Lie groups (for a fixed prime $p$ ) define an admissible property of locally compact groups. Similarly, the Lie groups (i.e., the Lie groups over the reals) define an admissible property of topological groups; the discrete groups do so by trivial arguments.

It should be mentioned that Lie groups are not assumed to be second countable in this text; in particular, any discrete group is a Lie group. Further examples of admissible properties of topological groups are given by the class of soluble locally compact groups, and also by the class of nilpotent locally compact groups.

2. Characterizations of pro-p-adic Lie groups. In this section, we prove several characterizations of locally compact pro-A-groups, where $\mathbb{A}$ is an admissible property of topological groups. As special cases, we obtain characterizations of locally compact pro- $p$-adic Lie groups, locally compact pro-discrete groups, and locally compact pro-Lie groups.

Theorem 2.1. Let $\mathbb{A}$ be an admissible property of topological groups, and $G$ be a locally compact group. Then the following conditions are equivalent:

(a) $G$ can be approximated by A-groups;

(b) $\mathrm{N}_{\mathbb{A}}(G)$ is a filter basis which converges to 1 in $G$;

(c) $G$ is a pro-A-group;

(d) $G$ is a $\mathbb{T} \mathbb{G}$-projective limit of $\mathbb{A}$-groups;

(e) $G$ is an element of the variety of Hausdorff groups $\mathcal{V}(\mathbb{A})$ generated by $\mathbb{A}$. 
Proof. Note that since $\mathbb{A}$ is an admissible property of topological groups and $G$ is locally compact, $\mathrm{N}_{A}(G)$ is a filter basis. The equivalence (a) $\Leftrightarrow(\mathrm{b})$, as well as the implications $(\mathrm{c}) \Rightarrow(\mathrm{d})$ and $(\mathrm{d}) \Rightarrow(\mathrm{e})$, are trivial.

(b) $\Rightarrow$ (c): If (b) holds, $\eta_{\mathbb{A}}$ is injective, since $\bigcap \mathrm{N}_{\mathbb{A}}(G)=\{1\}$; furthermore, $\eta_{\mathbb{A}}$ has dense image, as observed above. We show that $\eta_{\mathbb{A}}: G \rightarrow G_{\mathbb{A}}$ is open onto its image: then $\operatorname{im} \eta_{\mathbb{A}}$ will be complete, hence closed, hence all of $G_{\mathbb{A}}$; thus, $\eta_{\mathbb{A}}$ being an isomorphism, Condition (III) will hold for $G$ as observed above, and Condition (II) is satisfied by $G$ since $\mathbb{A}$ is an admissible property of topological groups. So let $U$ be an identity neighbourhood in $G$. Then there is an identity neighbourhood $V$ such that $V V \subseteq U$. Since $G$ is a pro-A-group, there is $N \in \mathrm{N}_{\mathbb{A}}(G)$ such that $N \subseteq V$. Then $V N \subseteq U$ is an $N$-saturated identity neighbourhood, and $\eta_{\mathbb{A}}(V N)=\operatorname{im} \eta_{\mathbb{A}} \cap$ $\operatorname{pr}_{N}^{-1}(V N / N)$ is open in im $\eta_{\mathbb{A}}$. Thus indeed $\eta_{\mathbb{A}}$ is an embedding.

$(\mathrm{e}) \Rightarrow(\mathrm{a})$ : Since $\mathcal{V}(\mathbb{A})=\mathrm{SC}(\mathbb{A})$, we may assume that $G$ is a subgroup of a product $\prod_{i \in I} H_{i}$ of $\mathbb{A}$-groups. If $U$ is an identity neighbourhood in $G$, there are a finite subset $F$ of $I$ and open identity neighbourhoods $U_{i}$ in $H_{i}$ for $i \in I$ such that $U_{i}=H_{i}$ for $i \in I \backslash F$ and $G \cap \prod_{i \in I} U_{i} \subseteq U$. Let $\operatorname{pr}_{F}: \prod_{i \in I} H_{i} \rightarrow H_{F}:=\prod_{i \in F} H_{i}$ be the projection and $f:=\left.\operatorname{pr}_{F}\right|_{G}$. Then $f: G \rightarrow H_{F}$ is a morphism into an A-group, whence $G / \operatorname{ker} f$ is an $\mathbb{A}$-group by admissibility of $\mathbb{A}$. Furthermore, $\operatorname{ker} f=G \cap \prod_{i \in I F} H_{i} \subseteq U$, as required.

3. A "pro-version" of Cartan's Theorem. Cartan's Theorem asserts that continuous homomorphisms between Lie groups over a common ground field $\mathbb{R}$ or $\mathbb{Q}_{p}$ are analytic [14, Part II, §V.9], that continuous homomorphisms between real and $p$-adic Lie groups (in either direction) are locally constant, and that so are continuous homomorphisms between $p$-adic and $q$-adic Lie groups if $p \neq q[3, \S 8.1$, Proposition 1]: in particular, a locally compact group is discrete if it is both a real Lie group and a $p$-adic Lie group, or both a $p$-adic Lie group and a $q$-adic Lie group. We now prove a "pro-version" of this classical fact.

TheORem 3.1. Let $G$ be a locally compact, pro-p-adic Lie group. If $G$ is also a pro-Lie group or a pro-q-adic Lie group for some prime $q \neq p$, then $G$ is pro-discrete.

Proof. Assume that $G$ is a pro- $p$-adic Lie group and a pro- $q$-adic Lie group, where $p \neq q$; the case in which $G$ is both a pro- $p$-adic Lie group and a pro-Lie group can be settled analogously. Let $U$ be an identity neighbourhood in $G$; then there is a compact, open subgroup $V$ of $G$ such that $V \subseteq U$. Since $G$ is a pro-p-adic Lie group and a pro- $q$-adic Lie group, there exist compact normal subgroups $K$ and $N$ of $G$ such that $G / K$ is a $p$-adic Lie group, $G / N$ is a $q$-adic Lie group, and $K \cup N \subseteq V$ (Theorem 2.1). Since $V$ is a group, we have $K N \subseteq V$; furthermore, $K N$ is a compact normal subgroup of $G$. Now $G / K N$, being both a quotient of $G / K$ and $G / N$, is both a $p$-adic Lie group and a $q$-adic Lie group. By Cartan's Theorem, $G / K N$ is discrete. We have proved that $G$ can be approximated by discrete groups; by Theorem $2.1, G$ is pro-discrete.

4. Approximation by $\mathbb{\llbracket} \mathbb{E}_{\mathfrak{p}}$-groups. In this section, we show that a locally compact group which can be approximated by $p$-adic Lie groups for variable primes $p$ is already a pro- $p$-adic Lie group for some prime $p$. 
In the following, $\mathbb{P}$ denotes the set of all primes; if $p$ is a prime, $\mathbb{L} \mathbb{a} \mathbb{E}_{p}$ denotes the class of $p$-adic Lie groups. We set $\mathbb{P}_{\infty}:=\mathbb{P} \cup\{\infty\}$ and let $\mathbb{\mathbb { Q }} \mathbb{} \mathbb{E}_{\infty}$ be the class of real Lie groups. If $\emptyset \neq \mathfrak{p} \subseteq \mathbb{P}_{\infty}$, we define $\mathbb{L} \mathbb{\mathbb { E } _ { \mathfrak { p } }}:=\bigcup_{p \in \mathfrak{p}} \mathbb{L} \mathbb{\mathbb { E }} \mathbb{E}_{p}$.

THEOREM 4.1. Suppose that $G$ is a locally compact group which can be approximated by $\mathbb{L} \mathbb{E}_{\mathfrak{p}}$-groups, where $\mathfrak{p}$ is a non-empty set of primes. Then $G$ is a pro-p-adic Lie group for some prime $p \in \mathfrak{p}$. If $G$ is not pro-discrete, the prime $p$ is uniquely determined.

Proof. Suppose that $G$ is not a pro- $p$-adic Lie group for any $p \in \mathfrak{p}$. Given an identity neighbourhood $U$ in $G$, there exists a compact open subgroup $V$ of $G$ such

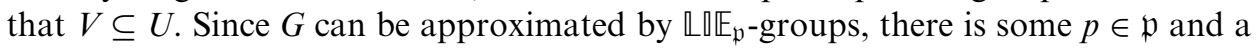
compact normal subgroup $K$ of $G$ such that $K \subseteq V$ and $G / K$ is a $p$-adic Lie group. Since $G$ is not a pro-p-adic Lie group, there exists an identity neighbourhood $W \subseteq V$ such that there is no compact normal subgroup $K^{\prime}$ of $G$ such that $K^{\prime} \subseteq W$ and $G / K^{\prime}$

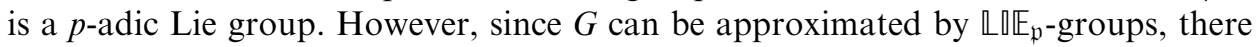
is $q \in \mathfrak{p}$ such that there exists a compact normal subgroup $N$ of $G$ such that $N \subseteq W$ and $G / N$ is a $q$-adic Lie group; by the preceding, $p \neq q$. Now $K N \subseteq V$ is a compact normal subgroup of $G$, and we deduce as in the proof of Theorem 3.1 that $G / K N$ is discrete. We infer that $G$ is pro-discrete. But then $G$ is a pro- $p$-adic Lie group for every $p \in \mathfrak{p}$, which is a contradiction. The remainder is clear in view of Theorem 3.1.

5. $\mathfrak{p}$-Mixtures. As a special case of Theorem 2.1, a locally compact group is a pro-p-adic Lie group if and only if it is a member of the variety of Hausdorff groups generated by the class $\mathbb{L} \mathbb{\mathbb { E } _ { p }}$ of $p$-adic Lie groups. If $q, r$ are distinct primes, then the product $Q \times R$ of a simple non-discrete $q$-adic Lie group $Q$ and a simple nondiscrete $r$-adic Lie group $R$ is a totally disconnected, locally compact group which is not a pro-p-adic Lie group for any prime $p$. It is rather obvious that if we try to build up more general totally disconnected, locally compact groups from $p$-adic Lie groups (like the preceding example), we should be allowed to mix different primes $p$. This motivates the following definition.

Definition 5.1. If $\emptyset \neq \mathfrak{p} \subseteq \mathbb{P}_{\infty}$, we set

$$
\begin{aligned}
& \mathbb{A}_{\mathfrak{p}}:=\overline{\mathrm{Q}} \overline{\mathrm{SP}}\left(\mathbb{L} \mathbb{\mathbb { E } _ { \mathfrak { p } }}\right),
\end{aligned}
$$



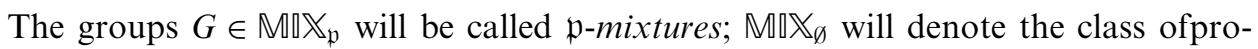
discrete, locally compact groups. We let $\mathbb{L}$ denote the class of all locally compact groups $L$ which can be made Lie groups over some local field (depending on $L$ ), and set $\mathbb{H}:=\overline{\mathrm{Q}} \overline{\mathrm{S}} \mathrm{P}(\mathbb{L})$.

Note that every $G \in \mathcal{V}\left(\mathbb{L} \mathbb{E}_{\mathbb{P}}\right)$ is totally disconnected, by Equation (1) above. By Theorem 2.1, a locally compact group $G$ is a $\{p\}$-mixture for a prime $p$ if and only if it is a pro-p-adic Lie group. We have already seen that the class of locally compact pro- $p$-adic Lie groups does not subsume all locally compact, totally disconnected groups. Although the class $\mathbb{M} \mathbb{\mathbb { X } _ { \mathbb { P } }}$ is much bigger, it still does not comprise all locally compact, totally disconnected groups, not even the compactly generated ones. 
THeOREM 5.2. There is a totally disconnected, locally compact group $G$ and a compactly generated, totally disconnected, locally compact group $H$ with the following properties.

(a) $G \notin \mathcal{V}(\mathbb{L})$, and $G$ has an open, normal subgroup $U$ such that $U \leq \operatorname{ker} f$, for every $f \in \operatorname{Hom}(G, \mathbb{H})$. In particular, $U \leq \operatorname{ker} f$ for all $f \in \operatorname{Hom}(G, \mathbb{L})$, and $G$ is not a P-mixture.

(b) $H$ is not a P-mixture, and $H$ has an open, normal subgroup $V$ such that $V \leq \operatorname{ker} f$ for every $f \in \operatorname{Hom}\left(H, A_{\mathbb{P}}\right)$.

Proof. We establish the theorem by means of three lemmas, the first of which is immediate from Equation (1) above.

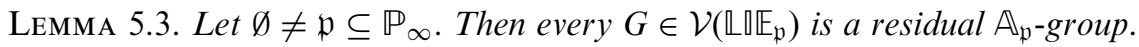

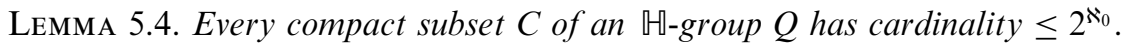

Proof. Assume first that $Q$ is a locally compact group which can be made a Lie group over some local field $K$. Being covered by finitely many balls, the compact subset $C$ of $Q$ has cardinality at most $2^{\aleph_{0}}$.

In the general case, $Q$ is a quotient of a closed subgroup $R$ of a product $P:=\prod_{i=1}^{n} S_{i}$ of $K_{i}$-Lie groups $S_{i}$ for certain local fields $K_{i}$. If $C$ is a compact subset of $Q$, we deduce from the local compactness of $R$ and the fact that $Q$ is a quotient of $R$ that the compact subset $C$ of $Q$ has a compact transversal $C^{\prime}$ in $R$. Set $C_{i}:=\operatorname{pr}_{i}\left(C^{\prime}\right)$. Then card $C_{i} \leq 2^{\aleph_{0}}$ for all $i$ by the above, and since $C^{\prime} \subseteq C_{1} \times \cdots \times C_{n}$, indeed card $C^{\prime} \leq 2^{\aleph_{0}}$. Hence card $C \leq 2^{\aleph_{0}}$ as well.

\section{Lemma 5.5. Every $A_{p}$-group A has a torsion-free, open subgroup.}

Proof. Up to topological isomorphism, any $\mathbb{A}_{\mathbb{p}}$-group $A$ has the form $A=S / N$, where $F$ is a finite set of primes, $G_{p}$ a $p$-adic Lie group for $p \in F, S$ a closed subgroup of $\prod_{p \in F} G_{p}$ (which we consider as an internal direct product), and $N$ a closed normal subgroup of $S$. We may assume that $\operatorname{pr}_{G_{p}}(S)$ is dense in $G_{p}$; then $N \cap G_{p}$ is a closed normal subgroup of $G_{p}$. After replacing $G_{p}$ with $G_{p} / N_{p} S$ with $S / \prod_{p \in F} N_{p}$, and $N$ with $N / \prod_{p \in F} N_{p}$, we may assume that $N \cap G_{p}=\{e\}$ for all $p \in F$. Let $H_{p}$ be a Campbell-Hausdorf (CH-) subgroup of $G_{p}$; i.e., an open compact subgroup which is topologically isom,orphic to an open $\mathbb{Z}_{p}$-submodel of the Lie algebra of $G_{p}$, equipped with the $\mathrm{CH}$-multiplication (cf. [3, §4.2, Lemma 3 and Theorem 2]); set $U:=\pi\left(S \cap \prod_{p \in F} H_{p}\right)$, where $\pi: S \rightarrow S / N=A$ is the quotient map. Then $U$ is an open, compact subgroup of $A$. It is torsion-free, since otherwise there exists $e / \neq x$ such that $x^{r}=e$ for some prime $r$. There is a continuous homomorphism $\xi: \mathbb{Z}_{r} \rightarrow S \cap \prod_{p \in F} H_{p}$ such that $\pi(\xi(1))=x \quad$ (cf. [6, Corollary 9.3]). Then $\operatorname{pr}_{G_{p}}(\operatorname{im} \xi)=\{\mathrm{e}\}$ for $p \neq r$, as $H_{p}$ is a CH-group, and thus $r \in F$ and $\operatorname{im} \xi \subseteq H_{r}$. Therefore $\xi\left(r \mathbb{Z}_{r}\right) \subseteq N \cap G_{r}=\{e\}$, entailing that $\xi(1) \neq e$ is an element of order $r$ in the $\mathrm{CH}$-group $H_{r}$. The latter being torsion-free, we have reached a contradiction.

We can prove Theorem 5.2 now.

Construction of $G$. Let $I$ be a set of cardinality $\operatorname{card}(I) \geq 2^{\aleph_{0}}$, and $p$ be a prime.

We consider the semidirect product $G:=\mathbb{Z}_{p}^{I} \times \operatorname{Sym}(I)$, where the group $\operatorname{Sym}(I)$ of all 
permutations of $I$ is equipped with the discrete topology and $U:=\mathbb{Z}_{p}^{I}$ carries the product topology. Then $G$ is a totally disconnected, locally compact group, and $U$ is an open, compact, normal subgroup. Suppose that $f \in \operatorname{Hom}(G, \mathbb{H})$; we claim that $U \leq \operatorname{ker} f$. Since $\operatorname{ker} f$ is closed, it suffices to show that $U \cap \operatorname{ker} f$ is dense in $U$. To see this, consider $x=\left(x_{i}\right) \in \mathbb{Z}_{p}^{I}$, and a finite subset $F$ of $I$; we show that there is $y=\left(y_{i}\right) U \in \cap \operatorname{ker} f$ such that $x_{i}=y_{i}$ for all $i \in F$. If $F$ has $n$ elements, say, we write $I=\bigcup_{\alpha \in I} I_{\alpha}$ as a disjoint union of sets $I_{\alpha}$ of cardinality $n$. Let $\operatorname{pr}_{\alpha}$ denote the projection of $\mathbb{Z}_{p}^{I}$ onto $\mathbb{Z}_{p}^{I_{\alpha}}$. Then there is some $\alpha \in I$ such that $N_{\alpha}:=\operatorname{pr}_{\alpha}(U \cap \operatorname{ker} f)=\mathbb{Z}_{p}^{I_{\alpha}}$. For if not so, set $Q:=\prod_{\alpha \in I} \mathbb{Z}_{p}^{I_{\alpha}} / N_{\alpha}$. Since $\left.f\right|_{U} ^{f(U)}$ is a quotient morphism due to compactness of $U$ and $U \cap \operatorname{ker} f \leq \prod_{\alpha \in I} N_{\alpha}=: N^{\prime}$, we deduce that $Q \cong U / N^{\prime}$ is a quotient of the compact group $f(U) \cong U / \cap$. Therefore card $f(U) \geq$ card $Q \geq 2^{\left(2^{\aleph_{0}}\right)}$, contradicting Lemma 5.4. Hence there is an $\alpha$ with the desired property. Pick a permutation $\phi$ of $I$ which maps $I_{\alpha}$ to $F$. Since $U \cap \operatorname{ker} f$ is a normal subgroup of $G=U \times \operatorname{Sym}(I)$, it follows from the definition of multiplication on the semidirect product that $U \cap \operatorname{ker} f$ is invariant under $\phi$. We deduce that $\operatorname{pr}_{F}(U \cap \operatorname{ker} f)=\mathbb{Z}_{p}^{F}$, where $\operatorname{pr}_{F}$ denotes the projection $\mathbb{Z}_{p}^{I} \rightarrow \mathbb{Z}_{p}^{F}$, as claimed. Thus $U \leq \operatorname{ker} f$ indeed. Since every member of $\mathcal{V}(\mathbb{L})$ is a residual $\mathbb{M}$-group by Equation (1) above, we deduce that $G$, not being a residual $\mathbb{H}$-group, is not a member of $\mathcal{V}(\mathbb{L})$.

Construction of $H$. Let $F$ be a non-trivial finite group, and $H:=F^{\mathbb{Z}} \rtimes \mathbb{Z}$, where $\mathbb{Z}$ acts on the compact group $V:=F^{\mathbb{Z}}$ by the shift action. Then every morphism $f: H \rightarrow L$ into an $A_{p}$-group $L$ has $V$ in its kernel. Indeed, let $W$ be a torsion-free, open subgroup of $L$. Then every torsion element in the open subgroup $f^{-1}(W)$ of $H$ is in the kernel of $f$. Since $V$ is a torsion group, $V \cap f^{-1}(W) \subseteq \operatorname{ker} f$, whence $\operatorname{ker} f \cap V$ is open in $V$. Note that $\operatorname{ker} f \cap V$ is a normal subgroup of $H$; using the shift action, it is easy to see that $\operatorname{ker} f \cap V$ is dense in $V$ and hence all of $V$ by closedness. In particular, $H$ is not a residual $\mathbb{A}_{\mathbb{p}}$-group and therefore not a $\mathbb{P}$-mixture, by Lemma 5.3 .

REMARK 5.6. Products of real and p-adic Lie groups occur naturally in the theory of algebraic groups over number fields (see [16]). For example, consider the field $\mathbb{Q}$ of rationals; then the set of places on $\mathbb{Q}$ can be parametrized by the set $\mathbb{P}_{\infty}$ defined above. Given a finite subset $S \subseteq \mathbb{P}_{\infty}$ such that $\infty \in S$, we set

$$
A_{S}:=\prod_{p \in S} \mathbb{Q}_{p} \times \prod_{p \in \mathbb{P} \backslash S} \mathbb{Z}_{p},
$$

where $\mathbb{Q}_{\infty}:=\mathbb{R}$. The union of the rings $A_{S}$, equipped with the direct limit topology, is the ring $A_{\mathbb{Q}}$ of adeles of $\mathbb{Q}$. If $G$ is an algebraic group over $\mathbb{Q}$, the Adele group of $G$ is the group $G\left(A_{\mathbb{Q}}\right)=\bigcup_{S} G\left(A_{S}\right)$, equipped with the direct limit topology, where

$$
G\left(A_{S}\right)=\prod_{p \in S} G\left(\mathbb{Q}_{p}\right) \times \prod_{p \in \mathbb{P} \backslash S} G\left(\mathbb{Z}_{p}\right)
$$

for finite subsets $S$ of $\mathbb{P}_{\infty}$ such that $\infty \in S$. Here $G\left(\mathbb{Q}_{p}\right)$ is equipped with its natural locally compact topology for $p \in S$, and $G\left(\mathbb{Z}_{p}\right)$ with its natural compact topology. Thus $G\left(A_{S}\right)$ is a locally compact group for all $S$, and so is $G\left(A_{\mathbb{Q}}\right)$, noting that $G\left(A_{\{\infty\}}\right)$ is an open subgroup of $G\left(A_{\mathbb{Q}}\right)$. Clearly $G\left(A_{S}\right)$ is an $S$-mixture for all $S$; however, one should not expect that $G\left(A_{\mathbb{Q}}\right)$ will be a $\mathbb{P}_{\infty}$-mixture in general. 


\section{Further examples and remarks.}

6.1 Every $p$-adic Lie group is a pro-p-adic Lie group. Every totally disconnected, compact group is pro-finite, hence pro-discrete; in particular, it is a pro- $p$-adic Lie group for every prime $p$, and also a pro-Lie group (cf. [8, Theorem 7.7]). Every totally disconnected, locally compact, abelian group $G$ is pro-discrete (loc. cit. Theorem 7.7). It is also known that any compactly generated, nilpotent, totally disconnected, locally compact group is pro-discrete [10].

6.2 The filter of zero-neighbourhoods of a locally convex $\mathbb{Q}_{p}$-vector space $V$ has a basis of open $\mathbb{Z}_{p}$-submodules of $V$ (see [13]); hence $\mathrm{N}_{\mathbb{D G}}(V)$ is a filter basis which converges to 0 in $V$. (Here $\mathbb{D G}$ denotes the class of discrete groups.) Proceeding as in the proof of Theorem 2.1, we find that $\eta_{\mathbb{D G}}: V \rightarrow V_{\mathbb{D G}}$ is an embedding; since Condition (II) is trivially satisfied, we easily deduce that $V$ is a pro-discrete group if and only if $V$ is complete.

6.3 (cf. [11, Example (0.3)]). We consider the multiplicatively written abelian group $V:=C_{3}^{(\mathbb{N})}$, equipped with the discrete topology. Since $\operatorname{Aut}\left(C_{3}\right) \cong C_{2}$ is compact, so is $C_{2}^{\mathbb{N}}$. Using the natural action of (Aut $\left.C_{3}\right)^{\mathbb{N}}$ on $V$, we obtain a semidirect product $G:=V \times C_{2}^{\mathbb{N}}$. Then $G$ is a totally disconnected, locally compact group which is a residually $p$-adic Lie group, since, for every $n \in \mathbb{N}$, we have a quotient morphism $G \rightarrow C_{3} \rtimes C_{2}$ with kernel $C_{3}^{(\mathbb{N} \backslash\{n\})} \rtimes C_{2}^{\mathbb{N} \backslash\{n\}}$. However, $G$ is not a pro- $p$-adic Lie group. To see this, note first that every identity neighbourhood of $G$ contains non-trivial torsion elements. Hence $G$ is not a $p$-adic Lie group. Now suppose that $G$ were a pro- $p$-adic Lie group. Then the compact, open subgroup $\{1\} \times C_{2}^{\mathbb{N}}$ would contain a compact, normal subgroup $N$ of $G$ such that $G / N$ is a $p$-adic Lie group. By the preceding, $N$ is not trivial, whence we find $(1, \alpha) \neq(1,1)$ in $N$. Algebraically, we may identify $G$ with a subgroup of $\left(C_{3} \rtimes C_{2}\right)^{\mathbb{N}}$. There is $n \in \mathbb{N}$ such that $\alpha(n) \neq 1$; therefore we find some $x \in V$ such that $\alpha(n) . x(n) \neq x(n)$ (the dot indicates the action of $C_{2} \cong \operatorname{Aut}\left(C_{3}\right)$ on $\left.C_{3}\right)$. Then $\left((x, 1)(1, \alpha)\left(x^{-1}, 1\right)\right)(n)=\left(x(n)(\alpha(n) \cdot x(n))^{-1}, \alpha(n)\right) \notin\{1\} \times$ $C_{2}$. Hence $(1, x)(\alpha, 1)(1, x)^{-1} \notin N$; that is, $N$ is not normal. This is a contradiction.

6.4 Being a residual $p$-adic Lie group (or a pro-p-adic Lie group) is not an extension property: indeed, the locally compact group $H$ constructed in the proof of Theorem 5.2 (b) is an extension of the pro-finite group $F^{\mathbb{Z}}$ by the discrete group $\mathbb{Z}$. See also the preceding example.

6.5 If $p$ and $q$ are distinct primes, we have $\mathbb{M} \square \mathbb{X}_{\{p\}} \cap \mathbb{M} \square \mathbb{X}_{\{q\}}=\mathbb{M} \square \mathbb{X}_{\{\emptyset\}}$ by

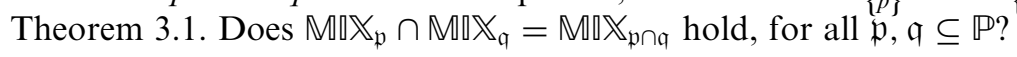

6.6 If $G$ is a totally disconnected, locally compact group, let $s_{G}: G \rightarrow \mathbb{N}$ be its scale function, as introduced in [17]. Let $\mathbb{P}(G)$ denote the set of primes occurring in the prime factor decompositions of the natural numbers $s_{G}(g)$, where $g \in G$; if $G$ is compactly generated, $\mathbb{P}(G)$ is a finite set [18]. If $s_{G} \equiv 1$, i.e., $\mathbb{P}(G)=\emptyset$, the group $G$ is called uniscalar; it is known that $G$ is uniscalar if and only if every $g \in G$ normalizes some open, compact subgroup of $G$. If $G$ is a locally compact, pro- $p$-adic Lie group,

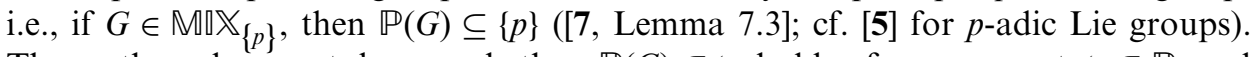
The author does not know whether $\mathbb{P}(G) \subseteq \mathfrak{p}$ holds, for every set $\mathfrak{p} \subseteq \mathbb{P}$ and $G \in \mathbb{M} \llbracket \mathbb{X}_{\mathfrak{p}}$. 
6.7 A compactly generated, locally compact, pro- $p$-adic Lie group $G$ is pro-discrete if and only if it is uniscalar ([7, Theorem 7.4]). However, there are compactly generated, totally disconnected, locally compact groups which are uniscalar but do not possess any compact, open, normal subgroup ([2], [12]).

Acknowledgements. The author wishes to thank Markus Stroppel (Stuttgart) for his suggestion that ideas from [11] might generalize to the $p$-adic setting.

\section{REFERENCES}

1. R. W. Bagley, T. S. Wu and J. S. Yang, Pro-Lie groups, Trans. Amer. Math. Soc. 287 (1985), 829-838.

2. A. Bhattacharjee and D. Macpherson, Strange permutation representations of free groups, Preprint, Leeds University, 1999.

3. N. Bourbaki, Groupes et Algebres de Lie, Chap. III (Hermann, Paris, 1972).

4. M. S. Brooks, S. A. Morris and S. A. Saxon, Generating varieties of topological groups, Proc. Edinburgh Math. Soc. (2) 18 (1973), 191-197. $205-215$

5. H. Glöckner, Scale functions on p-adic Lie groups, Manuscripta Math. 97 (1998),

6. H. Glöckner, Real and $p$-adic Lie algebra functors on the category of topological groups, Pacific J. Math., to appear. 413-421.

7. H. Glöckner and G. A. Willis, Uniscalar p-adic Lie groups, Forum Math., 13 (2001),

8. E. Hewitt and K. A. Ross, Abstract harmonic analysis I (Springer-Verlag, 1979).

9. K. H. Hofmann, Category-theoretical methods in topological algebra, in: Categorical topology, Lecture Notes in Math. No 540 (Springer-Verlag, 1976), 345-403.

10. K. H. Hofmann, J. R. Liukkonen and M. W. Mislove, Compact extensions of compactly generated nilpotent groups are pro-Lie, Proc. Amer. Math. Soc. 84 (1982), 443-448.

11. K. H. Hofmann, S. A. Morris and M. Stroppel, Locally compact groups, residual Lie groups, and varieties generated by Lie groups, Topology Appl. 71 (1996), 63-91.

12. A. Kepert and G. A. Willis, Scale functions and tree ends, J. Austr. Math. Soc. 70 (2001), 271-292.

13. A. F. Monna, Analyse non-Archimédienne (Springer-Verlag, 1970).

14. J.-P. Serre, Lie groups and Lie algebras, Lecture Notes in Math. 1500 (Springer-Verlag, 1992). $351-365$

15. H. Yamabe, A generalization of a theorem of Gleason, Ann. of Math. 58 (1953),

16. A. Weil, Adeles and algebraic groups (Birkhäuser, 1982).

17. G. A. Willis, The structure of totally disconnected, locally compact groups, Math. Ann. 300 (1994), 341-363.

18. G. A. Willis, The scale function on a compactly generated group has a finite number of prime divisors, Bull. London Math. Soc., to appear. 